\title{
Perfiles de participación en la red de los estudiantes universitarios y sus repercusiones en el aprendizaje
}

\section{Profiles of Participation in the Network of University Students and their Impact on Learning}

\author{
Marta López Costa \\ Universidad de Barcelona. España. \\ m.lopez@ub.edu
}

\begin{abstract}
Resumen
Vivimos en una sociedad digital que está en constante desarrollo tecnológico. La alta frecuencia de conexión que tienen los jóvenes en Internet ha provocado que en determinadas ocasiones se atribuya a esta generación un alto empoderamiento digital. Recientes estudios demuestran que no por conectarse habitualmente en Internet implica poseer altas competencias digitales (Kennedy, 2008, 2010; Gros, García y Escofet, 2012). Por este motivo, para realizar un buen diseño educativo a través del uso de tecnología emergente, resulta necesario analizar las prácticas en la red de los universitarios.

La investigación que se presenta pone en el punto de mira la voz del estudiante y su participación digital. El objetivo principal es analizar las prácticas socioculturales y los espacios digitales de participación en la red, en cualquier contexto, donde la tecnología puede actuar como mediadora del aprendizaje. La metodología se basa en la aplicación de un cuestionario elaborado para medir el perfil de participación en la red en distintos contextos (académicos, profesionales y personales). Ha sido respondido por 368 estudiantes de la Universitat de Barcelona y la Universitat Oberta de Catalunya.

Los resultados señalan una heterogeneidad de los perfiles de participación en la red de los estudiantes universitarios.
\end{abstract}

\section{Palabras clave}

Universidad, tecnología digital, participación en la red, empoderamiento digital, estudiantes

\begin{abstract}
We live in a digital society that has a permanent technological evolution. A high level of participation of young people in Internet has induced us to assume that young people have high digital skills. Recent studies show us that high Internet connection does not mean having high digital skills (Kennedy, 2008, 2010; Gros, Garcia and Escofet, 2012). For this reason, in order to create a good educational design through the use of emerging technology, it is necessary to analyze the practices of university network.

The following research puts the voice of the students and their digital participation in the spotlight. The main goal of this research is to analyze practices and digital spaces of participation in the network, where the technology acts as an intermediary for university student learning. The methodology used is based on a questionnaire designed to measure the profile of participation in the network, in different contexts (academic, professional and personal). 368 students from the Universitat de Barcelona and Universitat Oberta de Catalunya answered it.

The results indicate students have heterogeneous profiles of participation in the network.
\end{abstract}

\section{Key words}

University, digital technology, network participation, digital competences, students 


\section{Antecedentes}

Sociedad en red: Participación y nuevas prácticas.

Constantemente se hace referencia a la expresión "vivimos en la sociedad de la información y el conocimiento", pero tal y como apuntó Castells (2001), todas las sociedades anteriores también han tenido la información y el conocimiento como fuente de poder, riqueza y significado, aunque en diferente grado y contenido. De este modo, el elemento que diferencia la sociedad actual no es la información, sino el informacionalismo (Castells 2001), es decir, la manera en que tratamos con la información.

La información sigue siendo fuente de poder, pero con la llegada de la revolución tecnológica y digital, este concepto se entiende desde una perspectiva diferente, y este es el hecho diferenciador del resto de sociedades. De acuerdo con Castells (2001), esta nueva perspectiva sobre la información implica una capacidad auto-expansiva de procesamiento y de comunicación en términos de volumen, complejidad y velocidad, así como una flexibilidad de distribución mediante redes interactivas y digitalizadas.

Actualmente, la información puede ser difundida y compartida de forma exponencial, a través de las redes de contactos que cada individuo posee. Además, esta información no sólo puede ser compartida, sino que cualquier usuario puede crear de nueva y modificar el contenido existente. Actualmente, la mayoría de la información está digitalizada, y por lo tanto, cualquier usuario tiene la posibilidad de acceder a ella a través de la red.

Debemos matizar que en la red nos podemos encontramos información que ya existía previamente, y por lo tanto, solamente ha sido digitalizada, o información y conocimiento que ha nacido directamente en la red, fruto de las interacciones entre usuarios. Algunos autores (Levy,1997; Woolley, 2010) han denominado a este fenómeno inteligencia colectiva.

Es evidente que las tecnologías digitales se han introducido en nuestra sociedad, y no sólo esto, sino que lo han hecho con mucha fuerza, y cada vez más resultan ser invisibles en nuestras prácticas. La causa de todo esto es fácil de averiguar. Tanto la sociedad como la economía son y se basan en relaciones sociales, por lo tanto, la primera causa de esta impactante revolución digital es el cambio que han provocado sobre las relaciones sociales (Terceiro y Matias, 2001). Las tecnologías digitales han transformado la manera en que la gente se comunica e interacciona dentro de la sociedad.

Históricamente, las relaciones sociales tenían una gran importancia, pero estas se basaban únicamente en relaciones cara a cara. Por este motivo, los gobiernos centraban sus esfuerzos en potenciar las redes de infraestructuras de transporte que facilitaban la movilidad tanto de personas como de mercancías.

Actualmente, esta situación ha cambiado y las relaciones ya no sólo necesitan el transporte para producirse. Las tecnologías digitales han conseguido crear una nueva tipología de relaciones sociales que han roto las barreras espacio y tiempo (Terceiro y Matias, 2001). Estas nuevas relaciones se sitúan en la red, y por lo tanto, desde

Perfiles de participación en la red de los estudiantes universitarios y sus repercusiones en el aprendizaje. 
cualquier lugar y en cualquier momento se puede tener la posibilidad de interactuar con cualquier persona o grupo.

Tanto la expansión de la información como la aparición de nuevas maneras de interaccionar, ha provocado que los ciudadanos hayamos ampliado nuestras prácticas socioculturales en el mundo digital. Según el informe Telefónica (2015), durante estos últimos años han aumentado las actividades relacionadas con la búsqueda de información a Internet (36,9\%), vender y comprar en la red (20\%), acceder a la banca electrónica (13\%) y participar en redes sociales $(10,7 \%)$.

Jóvenes con empoderamiento digital heterogéneo.

La generación de jóvenes actual se ha visto influenciada por su relación con los nuevos medios digitales, así como por las nuevas redes de comunicación y los nuevos valores ante las transformaciones de la sociedad digital.

Debido a los cambios económicos, sociales, políticos y educativos que ha habido en la sociedad durante las últimas décadas, los jóvenes de hoy en día han adoptado nuevas prácticas sociales que han hecho distinguirlos de las generaciones anteriores.

Actualmente, según el informe INE (2014), un 32\% de los jóvenes residentes en España entre 12 y 18 años realiza actividades en Internet durante más de tres horas diarias, y un $17,5 \%$ lo hace menos de una hora. Los jóvenes son la generación con más conectividad.

Las primeras investigaciones y análisis que se realizaron entorno a la generación de jóvenes que habían nacido durante la revolución digital apuntaban a que la alta frecuencia de uso de determinadas tecnologías digitales implicaba un elevado empoderamiento de los jóvenes en relación al uso de las TIC (Prensky, 2001, Tapscoot, 1997, Oblinger, 2006). Estos autores llegaron a etiquetar los jóvenes como "Nativos digitales" (Presnky 2001) o "Net Gener" (Oblinger, 2006), refiriéndose a sus altas competencias digitales.

Pero, recientemente, diferentes estudios (Lenhart, Madden, MacGill y Smith, 2008; Kennedy, 2008, 2010; Bennet, Maton y Kervin, 2008; Gros, García y Escofet, 2012) han mostrado como la variable edad no determina el empoderamiento de los jóvenes. De este modo, no todos los jóvenes tienen un perfil homogéneo en relación a las competencias digitales ni a su uso.

White y Le Cornu (2011) han decidido romper con las etiquetas asignadas a los jóvenes "nativos digitales" relacionadas con su uso homogéneo y sus altas competencias digitales, y han creado unas nuevas etiquetas que no están relacionadas con la edad. Estos autores distinguen entre "visitantes" (usuarios sin una participación activa en Internet, y sin preocupaciones de gestionar una identidad digital) y los "residentes" (mantienen y desarrollan continuamente y activamente su identidad virtual).

Aunque no toda la generación joven tenga un perfil homogéneo en relación a sus prácticas socioculturales en Internet, se han detectado una serie de tendencias transversales que debemos destacar:

Perfiles de participación en la red de los estudiantes universitarios y sus repercusiones en el aprendizaje. 
Nuevas formas de crear, organizar y buscar la información.

Como acabamos de ver, una de las características principales de la sociedad actual es la gran cantidad de información disponible a través de la red, y el acceso inmediato que tienen los usuarios a toda esta información. A lo largo de la historia, la información ha estado al alcance de muy poca gente, tan sólo estaba disponible para aquellos que poseían poder: la iglesia, la nobleza, la burguesía, y más adelante por los medios de comunicación de masas.

Actualmente estamos delante una situación única, la información está al servicio de los ciudadanos, y además, podemos acceder a información que se puede reproducir en tiempo real.

Un claro ejemplo de esta instantaneidad y atemporalidad es la noticia y difusión de la muerte de Bin Laden a través de Twitter. Esta red social dio a conocer la muerte de Osama Bin Laden y rompió el récord de Tuits por segundo. Además, fue el primer medio donde se pudo leer la noticia. Este hecho hizo reconocer a los analistas de medios de comunicación que las noticias a través de este medio, confirmadas o no, son más accesibles y más instantáneas que nunca.

La gran cantidad de información y la instantaneidad ha provocado que los ciudadanos precisen de nuevas formas de organizar y buscar la información. Actualmente, los jóvenes no tienen la percepción de una necesidad de memorizar muchos contenidos, ya que Internet, con sus motores de búsqueda, facilita la información que se adapta a sus necesidades (Sparrow, Liu y Wenger, 2011).

Con el nacimiento de la web nació una forma de estructurar la información que fue una revolución, el hipertexto, una de las estructuras básicas de organización de la información en la actualidad. Un texto compuesto de bloques de palabras o imágenes electrónicamente unidos mediante múltiples trayectos, cadenas o recorridos en una textualidad abierta (Landow, 2009). En este texto el usuario puede navegar entre una galaxia de significados y acceder a través de varias vías.

Actualmente, siguen apareciendo nuevas formas de organización de la información, nacidas a través de las redes sociales. Un ejemplo es el Hashtag, un símbolo que nos ayuda a clasificar la información. A través del hash \# categorizamos la información que subimos a Internet. Además, las redes sociales pueden realizar un ranking de las categorías y temas más populares.

Por otro lado, la creación de contenidos es una de las prácticas en Internet que está en aumento. Actualmente no es tan frecuente como la búsqueda de información pero ya un $72,4 \%$ de los jóvenes españoles de entre 16 a 24 publican contenidos en Internet como por ejemplo fotografías, textos o vídeos (INE 2014). Los jóvenes tienen el porcentaje más elevado respecto al resto de franjas de edad. Las redes sociales son el lugar donde los jóvenes publican más contenido. Según el estudio de Madden, Lenhart, Cortesi, Gasser, Duggan, Smith, y Beaton (2013), el 71,6\% de jóvenes tiene un perfil en la redes sociales. Facebook es la red social más utilizada, aunque los usuarios de Twitter año tras año va aumentado.

Perfiles de participación en la red de los estudiantes universitarios y sus repercusiones en el aprendizaje. 
Tecnología ubicua.

Actualmente las tecnologías móviles y ubicuas están tomando un rol importante entre los jóvenes. Entendemos por dispositivos ubicuos aquella tecnología integrada en el entorno de la persona. Son diferentes autores (Norma, 1999; Hansmann, 2003) los que interpretan esta ubicuidad como una invisibilidad de los dispositivos tecnológicos, es decir, la adaptación completa de los ordenadores, móviles y tablets a las necesidades y formas de vivir de las personas.

Las conexiones fijas vinculadas a ordenadores fijos conviven ya con las conexiones móviles. Gracias, tanto al desarrollo de las pantallas multitáctiles, al diseño de las interfaces de respuesta inmediata con una navegación sencilla e intuitiva, como al desarrollo de la banda ancha móvil, la difusión de estos dispositivos ha sido muy rápida (Oró, M, Lanna, L y Casas, K, 2013).

Actualmente los dispositivos móviles, como los teléfonos inteligentes, nos permiten conectarnos con una gran facilidad desde cualquier lugar, ya sea esperando el transporte público, dentro de un bar o en medio de un parque.

El Informe Anual de la Sociedad en Red (2015) muestra como el correo electrónico, las redes sociales y la búsqueda de información son las prácticas más populares realizadas en Internet. En cambio, comunicarse a través de mensajería instantánea es la práctica más realizada a través del móvil. Seguidamente, nos encontramos el uso de redes sociales y el correo electrónico como las prácticas más frecuentadas con móvil.

Nuevos modelos de comunidad y relación en red.

La vinculación de personas por intereses o características comunes ha existido desde los inicios del ser humano. Las comunidades de personas se forman a partir del parentesco y las relaciones provenientes de puestos de trabajo, amistades, grupos de interés, lazos vecinales, entre otros, que contactan para formar redes que ofrezcan la sociabilidad, la ayuda, el apoyo y el control social (Reig y Martín, 2013).

Las tecnologías digitales han creado nuevos espacios de interacción entre personas. De este modo, no sólo tenemos la oportunidad de comunicarnos cara a cara y con grupos localizados geográficamente, sino que podemos interaccionar con comunidades virtuales, que rompen con las limitaciones geográficas y temporales.

Las personas se comunican y interaccionan en estas redes virtuales, en lugar de estar ligados a una sola comunidad. Sin embargo, debemos destacar que las redes de personas que siguen teniendo un número importante de interacciones son las familiares y vecinos, las comunidades tradicionales, así como la de los amigos y los compañeros de trabajo. Así lo muestra el estudio de Hampton y Wellman (2003) que pretende analizar, a través de una investigación etnográfica, como Internet afecta a las comunidades de vecinos. El informe concluye que Internet, como método de comunicación, tiene muchas ventajas sobre las formas de comunicación, puesto que facilita las interacciones de las comunidades tradicionales.

Perfiles de participación en la red de los estudiantes universitarios y sus repercusiones en el aprendizaje. 
Además, la utilización de herramientas digitales para interaccionar, es otro medio de comunicación (Sánchez, 2015). Y como tal, debemos entender que están definidas por las necesidades de cada etapa evolutiva de las personas, no están definidas por las potencialidades que ofrece la red.

"El uso cotidiano de las tecnologías digitales que realizan los adolescentes tiene que ver con sus necesidades y dinámicas sociales y culturales propias de su etapa evolutiva" (Sánchez, 2015, p.89).

Universidad: Prácticas de aprendizaje formales, no formales e informales.

Recientemente las barreras que marcaban las diferencias entre el aprendizaje formal e informal eran bastante claras, a pesar de que cada concepto podía tener matices diferentes dependiendo del autor que los definía. Actualmente esta situación está cambiando, puesto que están apareciendo nuevas prácticas de aprendizaje que resultan casi imposible situarlas exactamente en un tipo de aprendizaje u otro. Estos conceptos de aprendizaje formal, no formal e informal están quedando obsoletos.

Además, las TIC han potenciado la aparición de nuevos elementos y actividades de aprendizaje que se sitúan de forma transversal en estas clásicas categorizaciones de la educación, y por lo tanto, las barreras entre aprendizaje formal, no formal e informal son cada vez más difusas.

Gracias a la web 2.0 y la facilidad que esta ofrece en el intercambio de información y la comunicación entre personas, se han abierto nuevos espacios en la red que permiten a los usuarios aprender fuera de las instituciones educativas y lejos de los currículums.

De este modo, la web social ha multiplicado los lugares donde los ciudadanos pueden aprender, construir y compartir conocimiento.

"In particular, pedagogues have been rethinking the implications of the web 2.0 in extending environments for situated, informal education, and for addressing the blurring of the boundaries between personal, social spaces and formal learning contexts" (JISC, 2007, p.35).

Hall (2009) propone un modelo de integración del aprendizaje. Es un modelo que pone de relieve los vínculos entre los dominios personales y los públicos, como por ejemplo el aprendizaje social en las comunidades, en el medios de comunicación y en las TIC.

La educación debe ser entendida como la experimentación continua en el aprendizaje a través de una combinación, de lugares, tiempos, tecnologías y entornos sociales. Esto implica que el aprendiz debe personalizar su experiencia de aprendizaje, y por lo tanto ser autónomo en este proceso. (Attwell, 2007; Downes, 2010; Hase i Kenyon, 2010). Es una perspectiva que fomenta un aprendizaje autorregulado con habilidades críticas por parte del estudiante, y a través de una participación activa con distintas comunidades, tanto dentro de las aulas como comunidades exteriores.

Perfiles de participación en la red de los estudiantes universitarios y sus repercusiones en el aprendizaje. 


\section{Metodología}

Este articulo se centra en una parte de una investigación de tesis doctoral llevada a cabo a través de una metodología mixta. Concretamente, el artículo pretende abordar la primera parte de la investigación, la cuantitativa, la cual tiene el objetivo de recopilar información demográfica detallada y categorizar los estudiantes universitarios en los diferentes perfiles de participación en la red, que determinan su identidad digital.

\section{Cuestionario}

El desarrollo de esta investigación se ha realizado a través de la elaboración y aplicación de un cuestionario. La finalidad del cuestionario ha sido categorizar a los estudiantes en diferentes perfiles de participación e analizar las herramientas y espacios digitales que utilizan.

El cuestionario está dividido en diferentes categorías relacionadas con los perfiles de participación. El marco teórico del proyecto ha permitido identificar una serie de aspectos y criterios a partir de los cuales se configuran los perfiles de participación en la red. Se ha creado este instrumento teniendo en cuenta diferentes dimensiones que se muestran a continuación. Estas dimensiones han servido para construir los diferentes perfiles de participación.

Cada dimisión tiene asignado distintos ítems en forma de pregunta. Cada ítem tiene una puntuación, 1 punto mínimo y un máximo de 5 puntos. A partir de las puntuaciones obtenidas de los estudiantes se han formado 3 perfiles de participación: Alto, medio y bajo.

En la siguiente tabla podemos ver en que perfil se sitúa el estudiante según la puntuación que ha obtenido en el cuestionario.

\begin{tabular}{|l|l|}
\hline Perfil bajo & 63 a 101 puntos \\
\hline Perfil medio & 102 a 128 puntos \\
\hline Perfil alto & 129 a 182 puntos \\
\hline
\end{tabular}

Tabla 1: Puntuación de perfiles de participación

Las dimensiones del cuestionario están basadas en dos teorías referentes a los perfiles de participación en la red, por un lado la de White y Cornu (2009) y por otro lado la de Bernoff y Lii (2008).

La aportación que realizan White y Cornu (2009), con la categorización de residentes y visitantes, aporta una nueva conceptualización sobre la participación en la red, puesto que se entiende, no como una generación que ha nacido rodeada de TIC, sino como usuarios que desarrollan continuamente y activamente su identidad digital. Por lo tanto, la participación en la red depende de la actividad que cada individuo realice.

Perfiles de participación en la red de los estudiantes universitarios y sus repercusiones en el aprendizaje. 
En esta misma línea de categorización, Bernoff y Lii (2008) realizan otra aportación sobre los tipos de participación en la red. No sólo ven la participación como activa, los llamados residentes, y pasiva, los visitantes, sino que dentro de los usuarios activos existen diferentes grados de participación: los creadores, los críticos, los colectores, los usuarios y los espectadores. Según las diferentes actividades que realizan en la red los usuarios se pueden clasificar en una u otra categoría. Según estos autores, no significa lo mismo ser activo leyendo webs, blogs y buscando información, que ser activo creando páginas webs, blogs, aportando y compartiendo conocimiento.

Además, hay que añadir que el grado de participación en la red de un mismo usuario puede variar según la actividad que esté realizando.

De este modo, el cuestionario pretende recoger datos de las siguientes dimensiones:

- Tipo de dispositivos y conexión

- Ubicuidad

- Participación en comunidades

- Herramientas de creación de contenido

- Herramientas de búsqueda de contenido e información

- Herramientas de gestión y organización de información

- Herramientas de ocio

- Finalidad de la participación en la red

Finalmente, en la tabla que se presenta a continuación se puede observar un ejemplo de la transformación de una de estas dimensiones, concretamente la dimensión de herramientas de gestión y organización de información, en los ítems concretos a analizar y su conversión en las preguntas del cuestionario. Además, como se ha comentado anteriormente, cada pregunta tiene asignada una puntuación para clasificar a los estudiantes en los perfiles de participación.

\begin{tabular}{|c|c|c|c|c|}
\hline Dimensión & $\begin{array}{l}\text { Puntos } \\
\text { totales }\end{array}$ & Ítem & Pregunta cuestionario & $\begin{array}{l}\text { Punto } \\
\text { S }\end{array}$ \\
\hline \multirow{7}{*}{$\begin{array}{l}\text { Gestión y } \\
\text { organizació } \\
\text { n de } \\
\text { informació } \\
\text { n (10\%) }\end{array}$} & \multirow{7}{*}{7 a 35} & \multirow{7}{*}{$\begin{array}{l}\text { Herramientas } \\
\text { para la gestión de } \\
\text { la información } \\
(7,7 \%)\end{array}$} & $\begin{array}{l}\text { Frecuencia de uso de herramientas } \\
\text { de creación colaborativas (Drive, } \\
\text { Teambox...) }\end{array}$ & 1 a 5 \\
\hline & & & $\begin{array}{l}\text { Frecuencia de creación y/o } \\
\text { desarrollo de proyectos }\end{array}$ & 1 a 5 \\
\hline & & & $\begin{array}{l}\text { Frecuencia de uso de disco duro } \\
\text { virtual (Dropbox) }\end{array}$ & 1 a 5 \\
\hline & & & $\begin{array}{l}\text { Frecuencia de descargas } \\
\text { aplicaciones }\end{array}$ & 1 a 5 \\
\hline & & & $\begin{array}{l}\text { Frecuencia de gestiones } \\
\text { administrativa (matrícula } \\
\text { universidad, bancos) }\end{array}$ & 1 a 5 \\
\hline & & & Frecuencia agregadores & 1 a 5 \\
\hline & & & $\begin{array}{l}\text { Frecuencia uso del PLE (personal } \\
\text { learning enviroment) }\end{array}$ & 1 a 5 \\
\hline
\end{tabular}

Perfiles de participación en la red de los estudiantes universitarios y sus repercusiones en el aprendizaje. 


\begin{tabular}{|c|c|c|c|c|}
\hline & & Finalidad de uso & $\begin{array}{l}\text { Finalidad uso de disco duro virtual } \\
\text { (Dropbox) }\end{array}$ & - \\
\hline & & $\begin{array}{l}\text { herramientas } \\
(2,2 \%)\end{array}$ & $\begin{array}{l}\text { Finalidad uso herramientas de } \\
\text { creación colaborativas (Drive, } \\
\text { Teambox...) }\end{array}$ & - \\
\hline
\end{tabular}

Tabla 2. Desglose de la dimensión de gestión y organización de la información

Validez y fiabilidad

Para realizar un análisis de validez de contenido del cuestionario se ha llevado a cabo varias acciones. Primeramente se ha creado una tabla de fundamentación de las dimensiones con su correspondiente representatividad dentro del instrumento. Las principales dimensiones que se pretenden medir son: participación en comunidades, creación de contenido, búsqueda de información y gestión de contenido. Todas estas dimensiones tienen un grado de importancia similar en el cuestionario, puesto que representan entre un 10 y $22 \%$ cada una.

Otra acción que se llevó a cabo es el juicio de expertos. Concretamente seis expertos, cinco especializados en el contenido teórico y uno en metodologías cualitativas, analizaron el cuestionario y realizaron aportaciones y sugerencias.

También se realizó un análisis de fiabilidad a través del método de consistencia interna basado en la Alfa de Cronbach. Esta prueba permite estimar la fiabilidad de un instrumento a través de un conjunto de ítems que se espera que midan la misma dimensión teórica. El valor obtenido es 0,867 , por lo tanto un valor aceptable, puesto que a partir de 0,8 se considera válido.

\section{Muestra}

La población de este estudio está formada por estudiantes de dos universidades de Cataluña:

- Universitat de Barcelona (UB): 42.019 estudiantes de grado

- Universitat Oberta de Catalunya (UOC): 25.233 estudiantes de grado

La población total de este estudio es de 67.251 estudiantes.

Para calcular la muestra representativa en base a la población mencionada, se aplicó un riesgo de alfa de 0,05 (Nivel de confianza 95\%) y un error muestral de 0,55. La muestra mínima representativa de estudiantes es de 316. Aun así, finalmente, el cuestionario fue respondido por 368 estudiantes.

Por otro lado, se tuvo en cuenta la representatividad en la muestra de los dos ámbitos de estudio, los técnicos-naturales, y los humanísticos-sociales, puesto que estudios previos ya mencionados demuestran como todavía existe una pequeña rendija digital entre estudiantes de estas dos ramas. Es decir, los estudiantes de ciencias sociales tienen una

Perfiles de participación en la red de los estudiantes universitarios y sus repercusiones en el aprendizaje. 
frecuencia de conexión, con la mayoría de herramientas digitales, inferior que los estudiantes de ciencias técnicas. Por lo tanto, resulta conveniente tener en cuenta representativamente los dos grupos.

En la UB los estudiantes quedan repartidos entre los dos grandes ámbitos de conocimiento de la siguiente forma: tanto en la población como en la muestra $65 \%$ provienen del ámbito humanístico-social, y el 35\% del ámbito técnico natural. En la UOC el $74 \%$ provienen del ámbito humanístico-social, y el $26 \%$ del ámbito técnico natural.

\section{Análisis de los datos}

Los datos obtenidos a través del cuestionario han sido trabajados con dos programas diferentes, por un lado SPSS, un software de análisis estadístico que permite prever y generar informes estadístico descriptivos como por ejemplo diagramas de tendencias, y otros análisis estadísticos complejos.

Para el análisis de los datos del cuestionario con SPSS se han realizado las siguientes pruebas estadísticas descriptivas:

\section{- Frecuencias}

- Tablas de contingencia con el contraste de Chi-cuadrado, para analizar si existe correlación entre diferentes variables.

Por otro lado, se ha utilizado el software SPAD, un programa que permite implementar una estrategia de análisis adecuado al tratamiento exploratorio multivariante de grandes tablas de datos (Bécue y Valls, 2000).

Este software ha servido para realizar una clasificación de los perfiles de participación (altos, medios y bajos) en función de sus respuestas comunes. Este análisis caracteriza los grupos en función de las variables recogidas, si estas son estadísticamente significativas sobre el total con una probabilidad $\mathrm{p}<, 001$.

\section{Resultados}

Datos demográficos

Los datos sociodemográficos que se presentan en el siguiente apartado hacen referencia a la muestra presentada anteriormente, 368 estudiantes provenientes de la Universitat de Barcelona y la Universitat Oberta de Catalunya.

La muestra está compuesta de un 63\% de mujeres (246) y un 37\% de hombres (122). Las áreas de estudio en que se distribuyen los individuos de la muestra corresponde en un $30 \%$ de los estudiantes del ámbito técnico-natural y en un $70 \%$ del ámbito humanístico-social.

Perfiles de participación en la red de los estudiantes universitarios y sus repercusiones en el aprendizaje. 
Tal y como se ha especificado en la muestra, el 33\% proviene de la UOC y el $66 \%$ de la $\mathrm{UB}$, de este modo se garantiza una representatividad equivalente en la muestra de estas universidades en relación a la población.

Además, un $63 \%$ son mujeres y un $37 \%$ hombres. Concretamente dentro de la UOC un $49 \%$ son hombres y un $51 \%$ son mujeres, en cambio a la UB hay un porcentaje más elevado de mujeres, $79 \%$, respeto el $21 \%$ de hombres.

La mayoría de los estudiantes se sitúan en la franja de edad de los 18 a los 21 años, estos representan un 50\% de los estudiantes universitarios participantes.

\section{Conectividad}

Referente a la conectividad de los estudiantes, destacar que la mayoría de estudiantes se conectan entre 3 y 5 horas diarias $(45,8 \%)$. La conexión en Internet del resto de estudiantes queda repartida de forma bastante equitativa entre más de 12 horas, entre 9 y 12 horas, entre 6 y 8 horas, y entre 0 a 2 horas.

El lugar donde los estudiantes se conectan con más frecuencia es en casa, ya sea en el despacho, donde un $85,5 \%$ se conecta frecuentemente o siempre, o en lugares más informales, como el salón o el dormitorio, donde un 70,1\% se conecta frecuentemente o siempre.

Contrariamente, el lugar donde los estudiantes se conectan menos es en bares o restaurantes, un $67,8 \%$ no se conecta nunca o raramente.

Por otro lado, hay que destacar que la universidad es un espacio donde los estudiantes se conectan ocasionalmente, ya sea dentro del aula o en espacios informales como el bar. Aun así, hay que destacar que hay un $60,1 \%$ de estudiantes que no se conecta nunca en clase o raramente.

\section{Perfiles de participación}

Los estudiantes universitarios encuestados se sitúan mayoritariamente en un perfil de participación en la red medio.

En el siguiente gráfico se ve como el 25,9\% de los estudiantes universitarios se sitúa en perfil de participación alto. El mismo porcentaje de estudiantes tienen un perfil de participación bajo, en cambio, la mayoría de los universitarios, alrededor del 50\% tienen un perfil de participación en la red medio. 


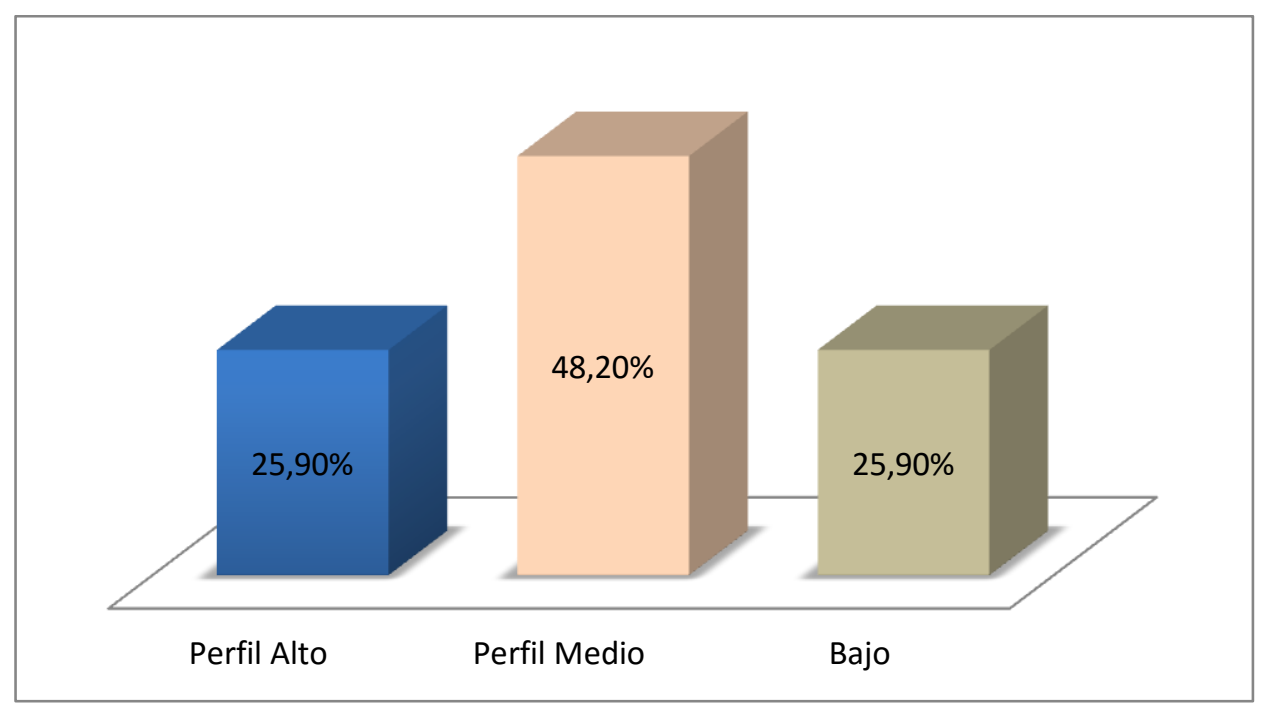

Gráfico 1: Perfiles de participación

Si realizamos un análisis concreto a los perfiles de participación bajos, vemos como se caracterizan por utilizar muy pocas herramientas digitales. Este grupo tiene como característica principal conectarse muy poco para realizar actividades en Internet, tan sólo para ver la televisión a través de Internet y para subir fotografías, actividades que hacen una vez al mes mínimo. Aparte, raramente utilizan los dispositivos móviles para conectarse en momentos que están en espera, como por ejemplo, en transporte público.

En cambio, el grupo de estudiantes con un perfil de participación medio es el grupo que tiene menos características en común. Los estudiantes con un perfil medio mantienen un uso de la tecnología diverso y heterogéneo. Las únicas características que tienen en común respecto a los otros dos perfiles son la no utilización de agregadores y el hecho de no participar en blogs.

Los estudiantes con perfil de participación alto conforman un grupo que comparte bastantes características. Estos se conectan a diario para ver vídeos, consultar información, comunicarse con sus contactos, leer blogs y compartir contenido. Es un grupo de estudiantes que utiliza diariamente o semanalmente la mayoría de las herramientas digitales, por tanto, una gran diversidad de herramientas, a diferencia de los otros perfiles de participación.

Finalmente, de forma general, destacar que las actividades más frecuentes entre los distintos perfiles de participación en la red son el envío de correo electrónico $(84,7 \%)$, la búsqueda de información (73,3\%), la mensajería instantánea $(81,7 \%)$, la participación en redes sociales $(55,9 \%)$ y la visualización de vídeos $(33,3 \%)$.

\section{Participación en comunidades}

Referente a la participación en comunidades, sigue habiendo una representatividad similar entre los diferentes perfiles de participación. Un 49,9\% de los estudiantes que tienen perfiles de participación bajos participan en comunidades, y un 59,1\% de los estudiantes con perfiles altos también participan. Por lo tanto, todos los perfiles mantienen un nivel de participación en comunidades similar. 
Las herramientas digitales clásicas para comunicarse en las diferentes comunidades siguen siendo las más utilizadas: el correo electrónico y la mensajería instantánea. Las redes sociales también son muy populares entre los estudiantes, un 56\% de los estudiantes las utiliza diariamente, y un $20 \%$ semanalmente. Otras herramientas como el microblogging (Twitter) no tienen tanta fuerza, puesto que un $66,2 \%$ de los estudiantes no las utiliza nunca o solamente alguna vez al mes.

La mayoría de las herramientas relacionadas con la participación en comunidad tiene una correlación significativa entre los diferentes perfiles de participación, es decir, los estudiantes con perfiles altos las utilizan con más frecuencia que los estudiantes con perfiles de participación bajos. Por ejemplo, el hecho de chatear y participar en las redes sociales tiene una correlación (Chi-cuadrado de 0,001) con los perfiles de participación.

\begin{tabular}{|l|l|}
\hline Perfil bajo & $30 \%$ \\
\hline Perfil medio & $56 \%$ \\
\hline Perfil alto & $65 \%$ \\
\hline
\end{tabular}

Tabla 4: Correlación redes sociales

Otro ejemplo es el caso de la participación en blogs, donde hay una correlación significativa $\mathrm{p}<0,05$ (concretamente de 0,000 ). Un $31 \%$ de los estudiantes con perfiles de participación altos realizan esta actividad semanalmente, mientras que tan sólo un $0,9 \%$ de los estudiantes con perfiles bajos realiza esta actividad. Y en el caso de la participación en foros también hay una correlación significativa $\mathrm{p}<0,05$ (de 0,000) donde solamente un $10,3 \%$ de estudiantes con perfiles bajos lo hace semanalmente o diariamente, frente al $55,2 \%$ de los perfiles altos que lo realiza semanalmente o diariamente.

Aun así, el envió de correos electrónicos es una actividad que no tiene una correlación significativa (Chi-cuadrado de 0,085 ) con los perfiles de participación, por lo tanto, y tal y como muestran los porcentajes, todos los perfiles envían correos con una frecuencia similar.

\begin{tabular}{|l|c|}
\hline Perfil bajo & $78 \%$ \\
\hline Perfil medio & $72 \%$ \\
\hline Perfil alto & $73 \%$ \\
\hline
\end{tabular}

Tabla 3: Correlación correo electrónico

Las actividades en Internet menos frecuentes tienen que ver con la ampliación de contactos a través de Internet. Sólo un $15,7 \%$ crea nuevos contactos semanalmente o

Perfiles de participación en la red de los estudiantes universitarios y sus repercusiones en el aprendizaje. 
diariamente, y un 32,7\% expone que nunca crea contactos nuevos a través de la red.

La participación en comunidades a través de blogs y wikis tampoco resulta ser muy frecuente, un $74,4 \%$ nunca participa en blogs, y un $81,7 \%$ nunca participa en wikis. Tan sólo un 7\% participa semanalmente o diariamente en blogs y un 4,6\% en wikis.

\section{Crear y gestionar contenido}

La creación de contenido es mucho más frecuente entre los usuarios de perfil alto que entre el resto de los perfiles.

El 35\% de los sujetos clasificados con un perfil alto de participación en la red realizan actividades relacionadas con la creación de contenido, en cambio, tan sólo un 14,2\% de los perfiles bajos realizan este tipo de actividad. La actividad que más realizan los estudiantes en relación a la creación de contenido es la elaboración de documentos de forma colaborativa, con herramientas como por ejemplo Google Drive.

El $44 \%$ de los estudiantes con perfiles de participación altos crean y comparten documentos semanalmente o diariamente, mientras que tan sólo un $16 \%$ de los estudiantes de perfil bajo lo hace. El hecho de compartir fotografías propias por parte de los estudiantes con perfil de participación alto, también se tiene que destacar, puesto que un $61 \%$ lo hace de forma diariamente o semanalmente. Los otros tipos de actividades, como mantener blogs personales semanalmente o a diario tienen una frecuencia de actividad muy inferior.

El disco duro virtual es una de las herramientas más utilizadas por los diferentes perfiles de participación en esta línea de actividades de gestión, y su relación de uso entre los perfiles resulta significativa. Por lo tanto, cuanto más alto es el perfil más alta es la probabilidad de frecuencia de esta herramienta. Casi el 50\% de los estudiantes de perfil alto utilizan este tipo de herramientas digitales. El porcentaje disminuye cuando se trata de los perfiles más bajos, a pesar de que se mantiene una correlación significativa no es una diferencia mucho elevada, tan sólo de un $17 \%$.

En cambio, otras actividades de gestión como la descarga de podcast, son muy poco frecuentadas por todos los perfiles de participación en general, sólo un $13 \%$ lo hace diaria o semanalmente, y la realización de gestiones administrativas, $24,5 \%$, tienen una frecuencia de uso mucho más baja por parte de todos los estudiantes.

\section{Búsqueda de información}

Los resultados referentes a la búsqueda de información tienen la misma tendencia que la de creación de contenido, es decir, cuanto más participativo en la red sea la estudiante más elevada es probabilidad de que realice búsquedas de información.

Entre los estudiantes de perfil bajo la búsqueda de contenido representa un 16,9\% entre todas las actividades que realizan en Internet, mientras que los de perfil alto la búsqueda de contenido representa un 30,8\% de su actividad en la red. Todas las actividades relacionadas con la búsqueda de contenido tienen una correlación significativa.

Perfiles de participación en la red de los estudiantes universitarios y sus repercusiones en el aprendizaje. 
La búsqueda de información a buscadores como Google es la actividad que más destaca, el resto son mucho menos frecuentes. En general, un 73,2\% de los estudiantes reconocen buscar información diaria o semanalmente. A pesar de haber una alta frecuencia por parte de los diferentes perfiles de participación, existe una correlación significativa. Por lo tanto, cuanto más alto sea el perfil de participación más alta será la probabilidad de que se busque información con más frecuencia.

Otras actividades como la consulta a enciclopedias $(15,8 \%)$, a fuentes de información académicas $(13,8 \%)$ o consultas a foros, tienen una frecuencia de uso diaria muy inferior $(3,4 \%)$. Aún así, hay que destacar que en todas ellas se mantiene la tendencia de que los perfiles con más participación en la red las realizan más a menudo.

\section{Finalidad académica}

La búsqueda de información es una de las actividades realizadas con más frecuencia para cualquier finalidad (académica o personal). Son un 93,5\% de los estudiantes que afirman realizar búsquedas de información para finalidades académicas. Todos los estudiantes, independientemente de su perfil de participación en la red, realizan actividades para finalidades académicas, aún así, los estudiantes con una participación en la red alta realizan muchas más actividades académicas en Internet y más diversas.

Las herramientas colaborativas, como por ejemplo el Google Drive, no tienen una frecuencia tan alta como las búsquedas de información, pero también hay un porcentaje bastante elevado de estudiantes que las utiliza para finalidades académicas, concretamente un $34,9 \%$, de los cuales un $25 \%$ de los estudiantes tienen un perfil de participación alto. En esta línea, un $64,9 \%$ de los estudiantes afirma compartir documentos para finalidades académicas.

Por otro lado, un 58,9\% de los estudiantes destaca que la mayoría de intervenciones en foros que realizan son para finalidades académicas. En cambio, referente a las redes sociales sólo participan un $27,2 \%$ de los estudiantes con este objetivo, de los cuales un $3 \%$ tienen un perfil de participación bajo.

Hay que destacar, que los estudiantes que miran contenido en formado vídeo a través de Internet para finalidades académicas tan sólo es un 37,6\%, la mayoría de los estudiantes lo hace para finalidades personales (un 83,3\%). El mantenimiento de webs y blogs para esta finalidad es también bastante bajo, pues no llega al $17 \%$ de los estudiantes que realiza esta actividad. La lectura de blogs tampoco consigue superar este 17\%. Los estudiantes prefieren consultar enciclopedias, en un $85,5 \%$.

\section{Finalidad personal}

Las actividades relacionadas con la finalidad personal son muy frecuentes entre los estudiantes. Del mismo modo que las actividades para finalidades personales, los estudiantes con perfiles altos de participación realizan más actividades para finalidades personales que los perfiles bajos. La mensajería instantánea, el correo electrónico y las videoconferencias son herramientas muy utilizadas para finalidades personales (un $89,1 \%, 93,7 \%$ y un $57,2 \%$ respectivamente).

Perfiles de participación en la red de los estudiantes universitarios y sus repercusiones en el aprendizaje. 
Las redes sociales también son herramientas de comunicación que predominan para este tipo de finalidad, un 83,9\% las utiliza a nivel personal. Además, el 59,9\% de los estudiantes afirma establecer contactos para finalidades personales. Por lo tanto, son bastantes las herramientas de comunicación empleadas para una finalidad personal, en comparación con las académicas.

La participación en blogs para finalidades personales también es bastante elevada, concretamente un 58,3\% de los estudiantes mantienen este tipo de participación, respeto el 16,3\% que leen blogs para finalidades académicas. Cabe destacar que de estos 58,3\% de los estudiantes, un $29 \%$ son de perfil alto. Aun así, la frecuencia disminuye cuando se habla de participación en blogs y wikis, que no supera el $20 \%$ entre todos los estudiantes.

Aparte de los blogs, los estudiantes (un 73,6\%) afirman leer diarios digitales para finalidades personales, mientras que solamente el $16,6 \%$ lo hace para finalidades académicas.

Hay que destacar también que la visión de contenidos en formado vídeo tiene que ver mayoritariamente con finalidades personales, un $86,9 \%$ de los estudiantes mira vídeos en Internet, ante el 37,6\% de los estudiantes que lo hacen por motivos académicos. La creación y el mantenimiento tanto de webs como de blogs es una acción realizada por muy pocos estudiantes, pero los pocos que lo realizan lo hacen para finalidades personales, sólo un 2,2\% para finalidades académicas.

\section{Discusión y conclusiones}

Los resultados de esta investigación apuntan en la línea de recientes investigaciones (Kennedy, 2008, 2010; Gordo, García y Escofet, 2012; Bennet, Maton y Kervin, 2008; Cheong, 2008), que señalan la heterogeneidad de los estudiantes universitarios en relación a la utilización de las TIC. Es decir, los estudiantes llegan a la universidad con diferentes competencias y usos de las tecnologías, tanto en el ámbito personal como en el académico.

Los universitarios participantes en la investigación se distribuyen mayoritariamente en el perfil medio (un 48,2\%), mientras que los perfiles altos representan un $25,9 \%$ y los bajos un $25,8 \%$. Por lo tanto, la variable de la edad no tiene una influencia determinada en la participación en la red de los estudiantes, puesto que esta es muy diversa en una misma generación, como es en este caso la de los jóvenes universitarios.

Si profundizamos en las características de cada uno de los perfiles de participación, los resultados nos muestran como, por un lado, los perfiles bajos, y por otra, los altos, comparten entre sí bastantes características relacionadas con la participación en la red, que a la vez son opuestas entre los dos grupos. Es decir, los miembros que forman parte del perfil bajo tienen características muy similares entre sí, así como los miembros que son de perfil alto, también tienen características similares entre ellos.

Perfiles de participación en la red de los estudiantes universitarios y sus repercusiones en el aprendizaje. 
En el caso de los perfiles altos, comparten características relacionadas con el uso de muchas herramientas digitales, conocen y utilizan una diversidad de prácticas en la red bastante elevada, es un perfil similar al que describieron Bernoff y Lii (2008) como usuarios creadores y críticos, es decir, los usuarios más activos en la red que se caracterizan para publicar contenido en webs y blogs, y comentar otros en páginas o en foros.

En cambio, en el caso de los perfiles bajos, la mayoría casi nunca se conectan en Internet para realizar actividades, tan sólo para mirar la TV a través de la red y para subir fotografías. Son un perfil cercano al concepto "visitantes" (White y Cornu, 2009), es decir, usuarios sin una identidad digital muy definida y bastante pasivos en la participación en la red que, por lo tanto, realizan una actividad invisible. Tan sólo utilizan las TIC como base de datos, no para pensar ni desarrollar ideas. También es un perfil similar al que Bernoff y Lii (2008) denominan como espectadores o inactivos, es decir que no realizan ninguna actividad o que simplemente leen blogs y webs pero no intervienen.

Los resultados de la presente investigación muestran como los perfiles altos se conectan diariamente para ver vídeos, consultar información, comunicarse con sus contactos y crear de nuevos, leer blogs y compartir contenido. Por lo tanto, sería un perfil cercano al concepto de "residentes" (White y Cornu, 2009), puesto que mantienen y desarrollan continuamente su identidad digital, y participan en las redes sociales y en la creación de contenido de una forma colaborativa. Aparte, utilizan plataformas más complejas, por ejemplo, la compra-venta o la elaboración propia de webs y blogs. Hay que destacar que ningún estudiantes ha sacado una puntuación máxima o cercana a la máxima, por lo tanto, existen perfiles altos pero no con una altísima participación en la red.

De este modo, ambos grupos entre sí son bastante heterogéneos. En cambio, los de perfil medio, donde se sitúa gran parte de los estudiantes, tienen características más dispersas entre sí. Las únicas características que tienen en común son la no utilización de agregadores ni tampoco la de participar en blogs. El resto de actividades en la red depende de cada estudiante.

La completa integración de las tecnologías por parte de los universitarios, adoptando esta perspectiva invisible, no se ha puesto de manifiesto en todos los ámbitos ni entre todos los estudiantes. Los universitarios participantes en la investigación se sitúan en diferentes perfiles de participación en la red, de este modo, la universidad y los docentes tienen que tener en cuenta esta diversidad de competencias y usos TIC por parte de los estudiantes a la hora de crear actividades o estrategias pedagógicas (Kennedy, 2008, 2010). Los diseños de las asignaturas tienen que contemplar que parte de los estudiantes pueden no tener los conocimientos mínimos para utilizar ciertas herramientas digitales, exceptuando el campus virtual, el Facebook o el Whatsapp.

Tan sólo las actividades relacionadas con la comunicación personal, es decir, con amigos y familia, son prácticas que se realizan de forma ubica y con una alta frecuencia. Para las prácticas, tanto personales, como académicas y profesionales, relacionadas con la creación, búsqueda y gestión del contenido e información, los jóvenes demuestran tener menos integradas las TIC. En todos los casos, el abanico de herramientas digitales es muy pequeño, y tan sólo se limita a herramientas como el campus virtual, el

Perfiles de participación en la red de los estudiantes universitarios y sus repercusiones en el aprendizaje. 
Whatsapp o redes sociales como el Facebook.

De este modo, los docentes pueden explotar esta característica de los estudiantes. A través de un diseño pedagógico se puede promover la interacción entre estudiantes fuera del aula, e incluso abrir las puertas a otros agentes, como comunidades ajenas o expertos externos, para realizar actividades de creación de contenido o de evaluación.

Las tecnologías digitales han multiplicado los espacios de interacción e intercambio entre las personas, haciendo llegar una gran cantidad de información y conocimiento, que anteriormente sólo estaba en manos de muy pocos, y teniendo el poder de crear y seguir compartiendo este conocimiento. Por lo tanto, no hay excusas para potenciar más que nunca un aprendizaje a lo largo de la vida, pero por eso, es necesario formar y educar a los jóvenes y a los educadores en la diversidad de herramientas y prácticas socioculturales digitales y en sus posibilidades por el aprendizaje integrado (Hall, 2009)

Además, las instituciones formales tienen que facilitar el uso de las TIC, tanto a nivel de espacios y equipamientos como nivel metodológico. En esta investigación, hemos visto cómo los estudiantes piden la integración de las tecnologías digitales de calidad, como por ejemplo el contenido audiovisual.

Para concluir, destacar que los estudiantes realizan determinadas prácticas digitales porque son arrastrados por la corriente de su entorno social. El mismo sucede en el ámbito académico. Los universitarios comentan que si el docente y las actividades de aprendizaje propuestas generaran esta necesidad no tendrían problema al realizar prácticas digitales. En algunos casos que ya ha sucedido los estudiantes lo han valorado muy positivamente. De este modo, si el estudiante se mueve en un contexto social y/o académico con un perfil de baja participación en la red es muy probable que reproduzca este mismo nivel, en cambio si se mueve dentro de un ambiente con perfiles altos se probable que también aumente su participación en la red.

Presentación del artículo: 13 de noviembre de 2015

Fecha de aprobación: 16 de marzo de 2016

Fecha de publicación: 30 de abril de 2016

López, M. (2016). Perfiles de participación en la red de los estudiantes universitarios y sus repercusiones en al aprendizaje. RED. Revista de Educación a Distancia. 49(10). Consultado el (dd/mm/aaaa) en http://www.um.es/ead/red/49

\section{Referencias}

Alfrey, L. (2010). The search for authenticity: How hipsters transformed from a local subculture to a global consumption collective. Washington: Georgetown University.

Bennett, S., Maton, K. y Kervin, L. (2008). The 'digital natives' debate: A critical review of the evidence. British Journal of Educational Technology, 39(5), 775-786.

Castells, M. (1996). The rise of the network society: The information age: Economy, society, and culture. Malden: Blackwell Publishers.

Perfiles de participación en la red de los estudiantes universitarios y sus repercusiones en el aprendizaje. 
Gros, B., Garcia, I. y Escofet, A. (2012). Beyond the net generation debate: A comparison of digital learners in face-to-face and virtual universities. International Review of Research in Open and Distance Learning, 12(4).

Kennedy, G., Judd, T., Churchward, A., Gray, K. y Krause, K. (2008). First year students' experiences with technology: Are they really digital natives. Australasian Journal of Educational Technology, 24(1), 108-122.

Kennedy, G. y Krause, K. (2007). Educating the net generation: Implications for learning and teaching in australian universities. Retrieved Mar, 28, 2007.

Kennedy, G., Dalgarno, B., Bennett, S., Gray, K., Waycott, J., Judd, T., et al. (2009). Educating the net generation: A handbook of findings for practice and policy.

Kennedy, G., Krause, K., Gray, K., Judd, T., Bennett, S., Maton, K., et al. (2006). Questioning the net generation: A collaborative project in australian higher education. Who's Learning, 413-417.

Lenhart, A., Kahne, J., Middaugh, E., Macgill, A. R., Evans, C. i Vitak, J. (2008). Teens, video games, and civics: Teens. Pew Internet \& American Life Project, 76.

Lévy, P. (1997). L'intelligence collective: pour une anthropologie du cyberspace. Paris: La Découverte.

Madden, M., Lenhart, A., Cortesi, S., Gasser, U., Duggan, M., Smith, A., y Beaton, M. (2013). Teens, social media, and privacy. Pew Research Center. Disponible a http://www.pewinternet.org/ /media//Files/Reports/2013/PIP_TeensSocialMediaand Privacy. pdf.

Negroponte, N. (2000). El mundo digital. Madrid: Suma de Letras.

Oblinger, D., y Oblinger, J. (2005). Is it age or IT: First steps toward understanding the net generation. Educating the net generation, 2(1-2), 20.

Oró, M. G., Lanna, L. C., y Casas, K. O. (2013). Cambios en el uso y la concepción de las TIC, implementando el Mobile Learning. RED. Revista de Educación a Distancia, (37), 1-19.

Prensky, M. (2001). Digital natives, digital immigrants part 2: Do they really think differently? On the Horizon, 9(6), 1-6.

Prensky, M. (2005). Listen to the natives. Educational leadership, 63(4).

Salinas, J. (1998). El rol del profesorado universitario ante los cambios de la era digital. Agenda Académica, 5(1), 131-141.

Smith, S. y Caruso, J. (2010). The ECAR study of undergraduate students and information technology, Boulder, CO: EDUCAUSE Center for Applied Research

Perfiles de participación en la red de los estudiantes universitarios y sus repercusiones en el aprendizaje. 
Sparrow, B., Liu, J. y Wegner, D. (2011). Google effects on memory: Cognitive consequences of having information at our fingertips. Science, 333(6043), 776-778.

Tapscott, D. (2009). Grown up digital: How the net generation is changing your world. New York: McGraw-Hill.

Terceiro, J. B., y Matías, G. (2001). Digitalismo. El nuevo horizonte socio.

White, D. i Le Cornu, A. (2011). Visitors and residents: A new typology for online engagement. First Monday, 16(9).

Woolley, A., Chabris, C. F., Pentland, A., Hashmi, N. i Malone, T. W. (2010). Evidence for a collective intelligence factor in the performance of human groups. Science, 330(6004), 686-688. 